\title{
Methods to Calibrate Oxygen Abundances of Star-Forming Galaxies and the Recent Results from the Large Sample of SDSS Galaxies
}

\author{
Y. C. Liang ${ }^{1}$, S. Y. Yin ${ }^{1,3}$, and F. Hammer ${ }^{2}$ \\ ${ }^{1}$ National Astronomical Observatories, Chinese Academy of Sciences, 20A Datun Road, \\ Chaoyang District, Beijing 100012, China; email: ycliang@bao.ac.cn \\ ${ }^{2}$ GEPI, Observatoire de Paris-Meudon, 92195 Meudon, France \\ ${ }^{3}$ Department of Physics, Hebei Normal University, Shijiazhuang 050016, China
}

\begin{abstract}
Using a large sample of $\sim 40,000$ star-forming galaxies selected from the SDSS, we derive oxygen abundance calibrations from strong-line ratios, such as $[\mathrm{N} \mathrm{II}] / \mathrm{H} \alpha,[\mathrm{O} \mathrm{III}] /[\mathrm{N} \mathrm{II}]$, $[\mathrm{N} \mathrm{II}] /[\mathrm{O} \mathrm{II}],[\mathrm{N}$ II $] /[\mathrm{S} \mathrm{II}],[\mathrm{S} \mathrm{II}] / \mathrm{H} \alpha$, and $[\mathrm{O} \mathrm{III}] / \mathrm{H} \beta$. The derived analytic calibrations cover a quite wide range of metallicity, from $12+\log (\mathrm{O} / \mathrm{H})=7.1$ to 9.3 . These calibrations can be used as calibration references for the future studies about metallicities of star-forming galaxies.
\end{abstract}

Keywords. galaxies: abundances, evolution, ISM, spiral, starburst

The basic methods to estimate oxygen abundances of star-forming galaxies are the $T_{e^{-}}, R_{23^{-}}, P$-method, and some other strong emission-line ratios, including $[\mathrm{N} \mathrm{II}] / \mathrm{H} \alpha$, [O $\mathrm{III}] /[\mathrm{N} \mathrm{II}],[\mathrm{N} \mathrm{II}] /[\mathrm{O} \mathrm{II}],[\mathrm{N} \mathrm{II}] /[\mathrm{S} \mathrm{II}],[\mathrm{S} \mathrm{II}] / \mathrm{H} \alpha$, and $[\mathrm{O} \mathrm{III}] / \mathrm{H} \beta$. We select 37,478 metalrich star-forming galaxies (with $12+\log (\mathrm{O} / \mathrm{H})>8.4$ ) from the SDSS-DR2, 531 metal-poor galaxies from the SDSS-DR4 with their [O III] 4363 detected at greater than $5 \sigma$, and 164 metal-poor galaxies and H II regions from literature with their $T_{e}$ measurements. Analytical abundance calibrations of the linear least squares and/or 3(or 2)-order polynomial fits from the strong-line ratios of these samples are obtained, which can be used as calibration references in the future studies. The observed relations are consistent with the photoionization models of Kewley \& Dopita (2002). Fig.1 shows the observed relations of the sample galaxies, the derived analytic calibrations, and the comparisons with models.
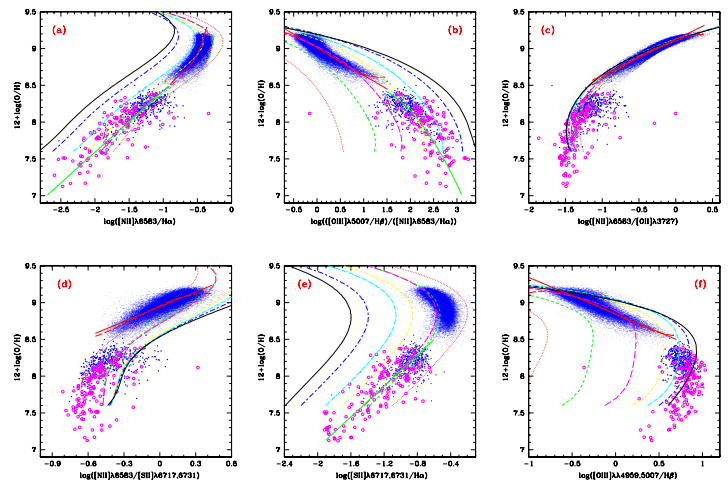

Figure 1. Analytic calibrations (linear least-squares and 3(or 2)-order polynomial fits) of strongline ratios for oxygen abundances from the large sample of SDSS galaxies (the blue points; the circles are from literature). The seven model lines are taken from Kewley \& Dopita (2002, ApJS, 142, 35). See Liang et al. (2006, ApJ, astro-ph/0607074) and Yin et al. (2006,A\&A, submitted) for more details. 